\title{
Heart Failure Patients With Ventricular Dysynchrony: Management With a Cardiac Resynchronization Therapy Device
}

\author{
Jean Flanagan, MSN, RN; ${ }^{1}$ Laura Horwood, RN; ${ }^{2}$ Cynthia Bolin, $\mathrm{RN} ;{ }^{3}$ Ross Sample, $\mathrm{RN}^{3}$
}

Despite an array of treatment modalities, the overall prognosis for patients with severe heart failure remains bleak. Biventricular pacing, or cardiac resynchronization therapy, is gaining increasing acceptance as a compelling treatment for those individuals with advanced heart failure (New York Heart Association functional class III or IV). This article provides a brief description of the atrial and ventricular conduction disturbances common in patients with advanced heart failure. Current indications for therapy are outlined, as are recent results of cardiac resynchronization therapy trials. The implant procedure is described to provide a comprehensive overview of this innovative approach to reestablishing normal electromechanical activity and synchronous right and left ventricular contractions. Patient care, before and after device implant, is also summarized. The focus on patient education throughout this article may allow it to serve as a reference for health care providers involved in the care of patients with severe heart failure. (Prog Cardiovasc Nurs. 2003;18:184-189) `2003 CHF, Inc.

From the Washington University Medical Center, St. Louis, MO; ${ }^{1}$ University of Michigan Medical Center, Ann Arbor, Ml; ${ }^{2}$ and the Guidant Corporation, St. Paul, $M N^{3}$

Address for correspondence:

Cynthia Bolin, RN, 939 Zohner Court,

St. Louis, MO 63031

E-mail: cynthia.bolin@guidant.com

Manuscript received March 25, 2003;

revised May 13, 2003;

accepted June 12, 2003

www.lejacq.com

ID: 2005
$\mathrm{H}$ eart failure (HF) is a major health concern that currently affects more than 5 million individuals in the United States. ${ }^{1}$ Approximately 500,000 new cases will be diagnosed each year. ${ }^{2}$ Morbidity remains high at nearly 300,000 deaths per year ${ }^{3}$ despite numerous pharmacologic advances in treatment. The cost of HF care has been estimated to be in excess of $\$ 11$ billion annually. ${ }^{4}$ Current treatment of HF includes medications, such as angiotensin-converting enzyme inhibitors, $\beta$ blockers, and aldosterone inhibitors, that have been proven to reduce mortality and hospitalizations. Heart transplantation and left ventricular assist devices can be offered to a relatively select group of individuals, and diuretics are utilized to reduce the symptoms associated with this syndrome. Nonpharmacologic strategies are aimed at providing comprehensive, ongoing disease management and promoting lifestyle modifications such as low sodium dietary intake, increased exercise, and smoking cessation.

Despite an array of treatment modalities, the overall prognosis for patients with severe HF remains bleak. Atrial-synchronized biventricular pacing, or cardiac resynchronization therapy (CRT), is gaining increasing acceptance as a minimally invasive, nonpharmacologic treatment for those individuals with advanced HF (New York Heart Association functional class III or IV).

\section{HF PATHOPHYSIOLOGY}

$\mathrm{HF}$ is the pathophysiologic state in which the heart is unable to maintain blood flow adequate to meet metabolic demands. Symptoms associated with HF can be attributed to abnormalities in contractile and hemodynamic function. In addition, a variety of atrial and ventricular conduction disturbances are noted in patients with advanced HF., ${ }^{5,6}$ The timing of atrial systole and ventricular activation can have an affect on diastolic filling times and presystolic mitral regurgitation. ${ }^{7}$ 
Interventricular conduction delays typically of a left bundle branch block morphology, can lead to mechanical dysynchrony further impairing cardiac function. ${ }^{8}$ With interventricular conduction delay, different portions of the ventricles contract and relax causing paradoxical wall motion and diminished contractile efficiency. Approximately $30 \%$ of all patients with advanced HF have a widened QRS complex indicative of some degree of intraventricular delay. ${ }^{9} \mathrm{~A}$ widened QRS complex is associated with increased mortality in the HF population ${ }^{10}$ (Figure 1).

\section{CRT}

\section{Pacing Therapy}

CRT aims to re-establish normal electromechanical activity. Recently, reports from clinical trials have shown that CRT improves functional status, exercise tolerance, and quality of life ${ }^{11}$ (Table I). The Comparison of Medical Therapy, Pacing and Defibrillation in Chronic Heart Failure (COMPANION) trial began in January 2000 in an effort to determine if CRT with optimal pharmacologic therapy, alone or in combination with backup defibrillation (CRT-D), could reduce all-cause mortality and hospitalization. At this writing, an independent data and safety monitoring board has recommended stopping the study due to achievement of this composite end point. ${ }^{12}$
CRT is accomplished using standard atrial and ventricular pacing leads. These leads are placed in the right atrium and right ventricle and used to pace the right side of the heart. New approaches have been developed to accomplish left ventricular pacing. A specially designed transvenous lead is inserted via the coronary sinus to a distal cardiac vein. The left ventricle is paced via this lead. This approach has been relatively successful and typically does not present a significant risk to the patient due to the lack of general anesthesia needs. In rare instances, like an inability to cannulate the coronary sinus, an epicardial lead is placed on the left ventricle via a thoracotomy or thoroscopy procedure. This is a more invasive procedure that requires the

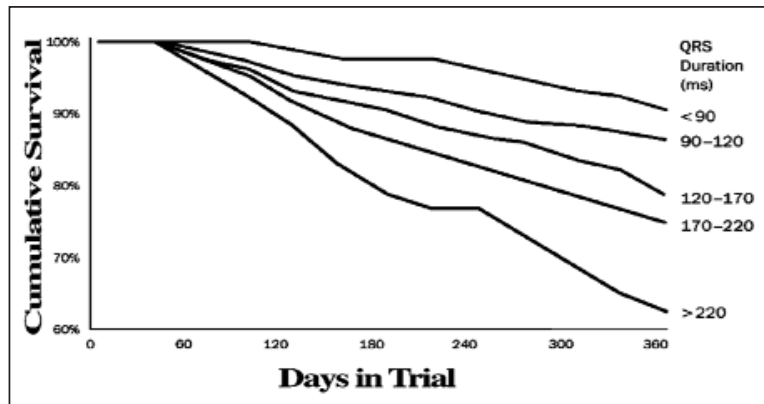

Figure 1. QRS duration and mortality. Reprinted with permission from Elseveir from J Allergy Clin Immunol. 1999;33:145A.

\begin{tabular}{|c|c|c|}
\hline StUdY & INCLUSION CRITERIA & RESULTS \\
\hline $\begin{array}{l}\text { Comparison of Medical } \\
\text { Therapy Pacing and } \\
\text { Defibrillation in Heart Failure } \\
(\text { COMPANION })^{12}\end{array}$ & $\begin{array}{l}\text { NYHA functional class III-IV, LVEF } \leq 35 \% \text {, } \\
\text { QRS } \geq 120 \mathrm{~ms}, \mathrm{PR}>150 \mathrm{~ms} \text {, no indication } \\
\text { for pacemaker or ICD }\end{array}$ & $\begin{array}{l}\text { Study stopped November } 2002 \text { due to } \\
\text { achievement of primary } \\
\text { end point }\end{array}$ \\
\hline $\begin{array}{l}\text { Pacing Therapies in } \\
\text { Congestive Heart Failure } \\
\text { (PATH-CHF) }\end{array}$ & $\begin{array}{l}\text { NYHA functional class II-IV, QRS }>120 \mathrm{~ms} \text {, } \\
\text { sinus rate }>55 \mathrm{bpm}\end{array}$ & $\begin{array}{l}\text { Improved functional status, } \mathrm{QOL} \text {, and } \\
\text { peak } \mathrm{VO}_{2} \text { status }\end{array}$ \\
\hline $\begin{array}{l}\text { VENTAK CHF/CONTAK } \\
\text { CD }^{14}\end{array}$ & $\begin{array}{l}\text { NYHA functional class II-IV, LVEF }<35 \% \text {, } \\
\text { QRS }>120 \text { ms, ICD indication }\end{array}$ & $\begin{array}{l}\text { Improvement in peak } \mathrm{VO}_{2} \text {, increase in } \\
6 \text {-minute walk distance, improved } \\
\text { functional status }\end{array}$ \\
\hline $\begin{array}{l}\text { Multicenter Insync } \\
\text { Randomized Clinical } \\
\text { Evaluation (MIRACLE) }^{15}\end{array}$ & $\begin{array}{l}\text { NYHA functional class III-IV, LVEF } \leq 35 \% \text {, } \\
\text { LVEDD } \geq 55 \mathrm{~mm} \text {, ORS } \geq 130 \mathrm{~ms} \text {; no pacing } \\
\text { indication; stable dosing of } \beta \text { blocker and } \\
\text { angiotensin-converting enzyme inhibitor }\end{array}$ & $\begin{array}{l}\text { Improved NYHA functional class, 6- } \\
\text { minute walk, QOL, LVEF, ventricular } \\
\text { volumes, mitral regurgitation, and peak } \\
\mathrm{VO}_{2} \text {; reduced hospitalizations }\end{array}$ \\
\hline $\begin{array}{l}\text { Cardiac Resynchronization in } \\
\text { Heart Failure (CARE-HF) }^{16}\end{array}$ & $\begin{array}{l}\text { NYHA functional class III-IV, LVEF } \leq 35 \%, \\
\text { LVEDD } \geq 30 \mathrm{~mm}, \text { QRS } \geq 150 \text { ms or } Q R S \geq 120 \\
\text { ms with defined echocardiographic criteria and } \\
\text { stable pharmacological therapy }\end{array}$ & Ongoing \\
\hline $\begin{array}{l}\text { Multisite Stimulation in } \\
\text { Cardiomyopathy Sinus } \\
\text { Rhythm (MUSTIC SR) }\end{array}$ & $\begin{array}{l}\text { NYHA functional class III, LVEF }<35 \% \text {, } \\
\text { LVEDD }>60 \mathrm{~mm} \text {, QRS }>150 \mathrm{~ms}, 6 \text {-minute } \\
\text { walk }<450 \text { feet }\end{array}$ & $\begin{array}{l}\text { Improved 6-minute walk, } \mathrm{QOL} \text {, and peak } \\
\mathrm{VO}_{2} ; \text { reduced hospitalizations; } 88 \% \text { of } \\
\text { patients preferred BiV pacing }\end{array}$ \\
\hline $\begin{array}{l}\mathrm{CRT}=\text { cardiac resynchronizatio } \\
\text { York Heart Association; } \mathrm{LVEF} \\
\mathrm{QOL}=\text { quality of life; } \mathrm{VO}_{2}=\mathrm{Oxyg}\end{array}$ & $\begin{array}{l}\text { rapy; } C R T-D=\text { cardiac resynchronization therap } \\
\text { ventricular ejection fraction; } P R=P R \text { interval; IC }\end{array}$ & $\begin{array}{l}\text { th backup defibrillation; } \mathrm{NYHA}=\mathrm{New} \\
\text { mplantable cardioverter defibrillator; } \\
\text { nd-diastolic diameter; } \mathrm{BiV}=\text { biventricular }\end{array}$ \\
\hline
\end{tabular}




\begin{tabular}{|c|c|}
\hline Table II. Indications for CRT and CR & \\
\hline CRT & CRT-D \\
\hline $\begin{array}{l}\text { Symptomatic despite stable, } \\
\text { optimal drug therapy }\end{array}$ & $\begin{array}{l}\text { Symptomatic despite stable, } \\
\text { optimal drug therapy }\end{array}$ \\
\hline $\begin{array}{l}\text { NYHA functional class III or IV moderate } \\
\text { to severe heart failure }\end{array}$ & $\begin{array}{l}\text { NYHA functional class III or IV moderate } \\
\text { to severe heart failure }\end{array}$ \\
\hline $\begin{array}{l}\text { Ventricular dysynchrony demonstrated } \\
\text { by a QRS duration } \geq 130 \mathrm{~ms}\end{array}$ & $\begin{array}{l}\text { Ventricular dysynchrony demonstrated } \\
\text { by a QRS duration } \geq 130 \mathrm{~ms}\end{array}$ \\
\hline $\begin{array}{l}\text { Severe left ventricle systolic dysfunction } \\
\text { evidenced by LVEF } \leq 35 \%\end{array}$ & $\begin{array}{l}\text { Severe left ventricle systolic dysfunction } \\
\text { evidenced by LVEF } \leq 35 \%\end{array}$ \\
\hline & $\begin{array}{l}\text { Patients at high risk of sudden cardiac death due to } \\
\text { ventricular arrhythmias: } \\
\text { Survival of at least one episode of cardiac arrest due to a } \\
\text { ventricular arrhythmia } \\
\text { Recurrent, poorly tolerated sustained VT } \\
\text { Prior MI, LVEF of }<35 \% \text {, and a documented episode of } \\
\text { nonsustained VT with an inducible ventricular } \\
\text { tachyarrhythmia. }\end{array}$ \\
\hline $\begin{array}{l}\text { CRT=cardiac resynchronization therapy; } \\
\text { NYHA=New York Heart Association; LV } \\
\text { infarction }\end{array}$ & $\begin{array}{l}\text { esynchronization therapy with backup defibrillation; } \\
\text { ejection fraction; VT=ventricular tachycardia; MI=myocardial }\end{array}$ \\
\hline
\end{tabular}

administration of a general anesthetic agent. With the three leads in place, the patient can receive traditional pacing therapy for bradycardic conditions along with CRT for optimal timing between atrial and ventricular contractions, improved coordination of the left ventricular septal, and free wall and coordinated contractile efforts of the left and right ventricular walls.

\section{Pacing and Defibrillation Therapy}

Mortality in HF patients may be due to end-stage HF as well as to sudden cardiac death (SCD). HF patients experience SCD at six to nine times the rate of the general population. ${ }^{2}$ Because the efficacy of the defibrillator (implantable cardioverter defibrillator [ICD]) in treating ventricular tachyarrhythmias and preventing SCD is well documented, this function has been included in some CRT devices. Clinical trials have been designed to study both CRT and CRT-D. Complete analyses of the COMPANION trial data may broaden the indications for ICD therapy and further define the advantages of combining these therapies.

\section{CRT PATIENT MANAGEMENT Patient Selection}

Criteria to identify appropriate candidates for CRT and CRT-D are based on the experiences gained during clinical trials (Table II). Trials to date have predominantly included patients with normal atrioventricular conduction and sinus node function. The benefit of CRT therapy in patients who require atrial pacing due to chronotropic incompetence or patients with atrial fibrillation has not been studied in large, double-blind, prospective clinical trials.

\section{Preoperative Preparation}

Patients undergoing implantation of a CRT device require comprehensive, preoperative evaluations to reduce patient risk and increase implant success. All efforts should be made to optimize the patient's cardiac status on recommended pharmacologic therapies before referral for device implant. A recent evaluation of cardiac performance (e.g., echocardiogram, cardiac catheterization) will be needed by the implanting physician to quantify atrial and ventricular chamber sizes, valve function, and left ventricular ejection fraction. A 12-lead electrocardiogram will identify abnormal atrioventricular conduction and widened QRS complexes documenting ICDs. Anticoagulation therapy considerations must be communicated to the implanting physician and/or professional nursing staff. Patients with a prosthetic valve may require continued anticoagulation before the procedure.

A thorough physical assessment is needed before device implant. Patients with advanced HF are at a higher risk for serious adverse events during and after the device implant. All efforts should focus on minimizing the inherent risks associated with prolonged intraoperative and postoperative bedrest, surgical blood loss, and anesthesia and contrast medium administration. Blood pressure, pulse, temperature, and pulse oximetry should be monitored. Patients must be well hydrated but without signs and symptoms of acute volume overload. A chest $\mathrm{x}$-ray can identify acute pulmonary vascular congestion requiring preoperative management. Standard laboratory analyses include a complete blood count, serum chemistry profile, coagulation studies, and urinalysis. Abnormal 
results should be immediately communicated to the implanting physician. Electrolyte imbalances should be corrected before the procedure. For CRT-D patients, hypokalemia or hyperkalemia may complicate the induction and successful termination of ventricular tachyarrhythmias during defibrillation threshold testing. Patients with abnormal renal function may require decreased or diluted contrast medium dosing.

Implanting physicians and professional support staff should be prepared to provide patients with complete explanation of CRT or CRT-D, risks and benefits, procedure length, multidisciplinary team members, anesthesia requirements, leads and device placement, postoperative observation, pain management, and follow-up care. Patients and their families must have ample time to review the information provided to them and to formulate questions. A member of the multidisciplinary team should be readily available to address concerns and provide support throughout the hospital stay.

\section{Implant Procedure}

Implantation of the CRT or CRT-D device can be done in either the cardiac catheterization laboratory or operative suite. Hospital policy, physician preference, staff competency, and accessibility of a fluoroscopy system will determine procedure location.

Procedure times can be long and will depend on physician experience level and patient anatomy. Reported procedure lengths vary from 1.5 hours to in excess of 5 hours. Significant time and effort may be spent on coronary sinus cannulation and left ventricular lead placement.

Conscious sedation may be provided for pain control or a patient may be intubated and general anesthesia used. If conscious sedation is used, it is imperative that the patient is adequately sedated to ensure a pain-free procedure and to minimize movement during the coronary sinus cannulation and left ventricular lead placement.

The implant techniques for CRT and CRT-D are similar to those utilized for standard pacemaker and defibrillator devices. The ventricular pacing or defibrillator lead is placed in the right ventricular apex or septum through the subclavian or cephalic vein. The atrial lead is placed in the right atrial appendage or elsewhere within the right atrium if the atrial appendage was damaged or removed during previous cardiac surgery.

The techniques and tools used for placement of the left ventricular lead are similar to those employed during an angioplasty procedure. A guide catheter is fed through a subclavian or cephalic vein to the right atrium. A steerable electrophysiology mapping catheter or guidewire is advanced through the guide catheter and into the coronary sinus ostium. The mapping catheter or wire is removed leaving the guide catheter in the coronary sinus ostium. A balloon catheter can be placed in the coronary sinus via the guide catheter. With the balloon inflated,

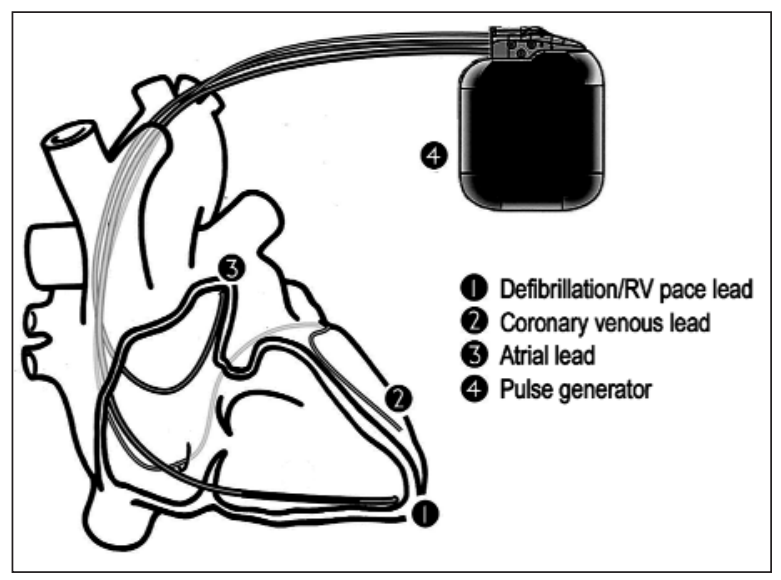

Figure 2. Anatomic illustration of the right atrial, right ventricular, and left ventricular pacing lead placement.

contrast medium is injected into the coronary sinus to visualize the branches of the coronary venous anatomy and facilitate vein selection for left ventricular lead placement. The left ventricular lead is then advanced to the target vein through the guide catheter (Figure 2).

Data from clinical trials suggest that vein selection may ultimately impact hemodynamic responses to left ventricle stimulation. Target veins include those that reach the midlateral wall of the left ventricle. Alternate veins can include the posterolateral and anterolateral branches. ${ }^{18}$ Left ventricular pacing can induce diaphragmatic stimulation and may affect lead position. Patient anatomy may limit placement options.

Atrial and right and left ventricular leads are tested during the procedure to verify adequate sensing capabilities and stimulation thresholds. The device is connected to the leads and placed within the pacemaker pocket. The wound is closed using standard surgical techniques. A sterile dressing is applied to the incision site. Additional testing is required for patients receiving a CRT-D device. Ventricular arrhythmias are induced to verify the defibrillator response and effective rhythm conversion.

Complications are rare but do occur during the implantation of a CRT or CRT-D device. A pneumothorax can result from attempts to gain initial subclavian vein access. Dissection or perforation of the coronary sinus can lead to cardiac tamponade. Left ventricular lead dislodgement can occur and often requires a second procedure for repositioning.

\section{Acute Postprocedure Care}

Postprocedure care for CRT-D patients can vary due to individual physician and/or hospital practice. General practice guidelines are outlined here.

Postprocedure telemetry monitoring is required to observe and evaluate device function. Pacemaker spikes may not be seen due to the routine use of bipolar atrial and right ventricular leads (Figure 3). Traditional single-chamber pacing and defibrillation devices provide 

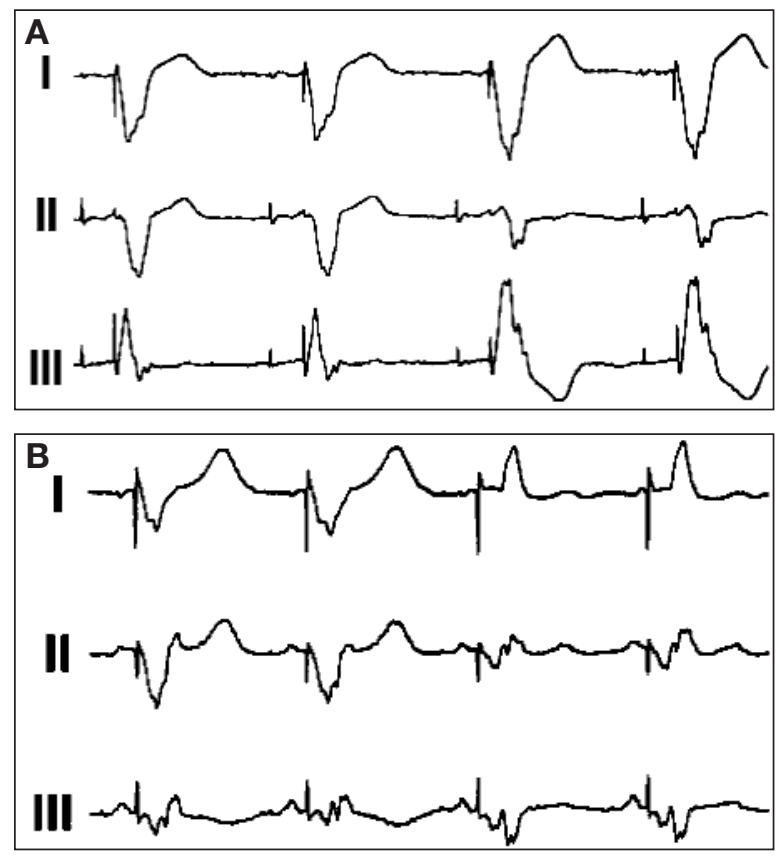

Figure 3. A) Biventricular to left ventricular only pacing. Vector changes noted in leads I and III associated with right ventricular loss of capture. B) Biventricular to right ventricular only pacing. Vector changes noted in leads I and III associated with left ventricular loss of capture.

ventricular pacing if the patient's rate falls below a set lower rate limit, so a combination of sensed and paced beats may be observed. The CRT and CRT-D devices are programmed to provide atrial synchronous pacing in both ventricles at all times. Current devices are equipped with multiple features that can be utilized to optimize resynchronization pacing and defibrillation therapies. These features can affect the pacing timing cycles and make rhythm interpretation difficult. Technical advice may be available by contacting the device manufacturer.

Patients receiving CRT devices may have an echocardiogram done before discharge from the hospital. The timing between atrial and ventricular contractions can be adjusted by utilizing device programming parameters. This procedure, referred to as atrioventricular optimization can be used to reduce mitral regurgitation and maximize left ventricular diastolic filling, resulting in improved cardiac output.

Generally, patients will remain on bedrest until the effects of the conscious sedation or general anesthesia have diminished. A sling may be used to temporarily immobilize the affected appendage. This can usually be removed for short periods while bathing or during a chest $\mathrm{x}$-ray. Patients are encouraged not to lift the arm on the implanted side above head level for at least 8 weeks. This may reduce the likelihood of lead dislodgement due to excessive pulling on the leads. Patients are also instructed to avoid lifting objects greater than 5-10 $\mathrm{lb}$ for the same period of time. A chest $\mathrm{x}$-ray obtained before discharge can identify lead movement and doc- ument final lead position. Diaphragmatic stimulation, absent during the testing phase of the procedure, may now become evident due to changes in patient positioning. The patient may complain of feeling the pacing pulses and/or prolonged hiccoughing. A physician should be notified of these findings. Noninvasive programming of the device frequently resolves this situation, but lead repositioning may be required.

A dressing or protective barrier is usually required for the first 24 hours. The dressing will be removed before discharge, but the wound should remain clean and dry for at least 1 week. Empiric antibiotic therapy may be given before and after the procedure.

The device may be evaluated before hospital discharge using a programmer and telemetry wand. This noninvasive testing is used to ensure that the device is accurately sensing atrial and ventricular activity. Stimulation threshold testing in the atrium, right ventricle, and left ventricle will be repeated to identify changes from implant values. Because pacing rates and electrocardiogram complexes can change during testing, the monitor observer should be notified before conducting this procedure. This predischarge evaluation will take approximately 15-20 minutes.

Swelling with or without a small hematoma formation at the implant site is quite common. If a large hematoma causes stress on the suture line, the implanting physician should be notified immediately. Blood coagulation levels should be evaluated closely and anticoagulation discontinued if appropriate.

Patients should be encouraged to communicate any postprocedure pain to health care providers. Oral analgesics will often alleviate the minor discomfort experienced during recovery from this procedure. ${ }^{19}$ (personal communication, Washington University Medical Center, Division of Cardiology Arrhythmia Services, St. Louis, MO)

\section{Discharge Education}

Patients and their families have numerous questions after device implant. Many of them do not arise until after discharge. Information packets are frequently used to reinforce patient education efforts. Device manufacturers provide patient education booklets and temporary identification cards. The card identifies the implanted leads and device and will often provide the patient with contact persons.

Patients commonly have concerns regarding driving, bathing, and wound care. To protect the leads from dislodgement, physicians will frequently restrict driving for at least 2 weeks. Some states prohibit driving for a period of 6-12 months after implantation of a defibrillator device. Restrictions on bathing will differ among implanters. Typically, patients are allowed to shower several days after implant but are instructed to gently cleanse the affected area and pat dry. Steri-strips applied to the incision site will gradually fall off within 
1-2 weeks. In the first several weeks after implant, the pocket may appear to be fluid filled. Gradually, the body will reabsorb the fluid. Depending on the size of the patient and pectoral muscle mass, the device may be quite visible to the observer. In larger patients, the device will be well hidden. Patients should be advised to follow all the standard restrictions outlined for patients with pacemakers or defibrillators. Infrequently, electromagnetic interference both in and out of the hospital setting can affect device performance.

CRT has been shown to improve the functional status of HF patients. ${ }^{15}$ Before discharge, patients should be encouraged to make a mental note of their current physical limitations. For example, in the weeks before the implant, was the patient able to climb a flight of stairs, sleep on only one pillow, or walk around the block? Patients will be asked to describe their current activity tolerance at subsequent follow-up visits. Some patients may not notice an immediate or dramatic response to therapy. It is important that they recognize even minor improvements in their quality of life.

Device manufacturers recommend device interrogation every 3 months to assess appropriate sensing and pacing performance. ${ }^{19,20}$ Patients with CRT and CRT-D devices may require additional visits due to the complexity of the underlying cardiac condition and its treatment. Medication changes and electrolyte imbalances may affect device performance. The CRT and CRT-D devices store recordings of atrial and ventricular arrhythmias. Arrhythmic events resulting in defibrillation or antitachycardia pacing can be analyzed for appropriateness of therapy.

The patient should understand the importance of regular follow-ups with medical personnel capable of performing device evaluations. The prevention or early diagnosis of potential system problems can be accomplished during these visits. Battery longevity of these devices is generally $3-6$ years, ${ }^{19,20}$ but this varies slightly by manufacturer. Battery longevity can be influenced by the frequency of arrhythmias requiring shocks and the amount of energy required to pace the myocardium. Patients should continue to be monitored by their cardiologist or HF specialist. Diuretic therapy dosing may need to be adjusted as a result of improved renal perfusion. Patients on low-dose $\beta$-blocker and vasodilator therapy may tolerate increased dosing because of the improved hemodynamic status associated with resynchronization therapy.

\section{SUMMARY}

Patients with advanced HF can remain symptomatic despite optimal pharmacologic and nonpharmacologic therapy. CRT has been shown to improve functional status and quality of life in a significant number of these individuals. The effect of CRT on mortality and hospi- talizations will emerge with final analyses of the COMPANION trial. All clinicians involved in the management of HF should be aware of this promising treatment for what continues to be a devastating disease.

\section{REFERENCES}

1 O'Connell JB. The economic burden of heart failure. Clin Cardiol. 2000;23(3 suppl):III6-III10.

2 American Heart Association. 2001 Heart and Stroke Statistical Update. Dallas, TX: American Heart Association; 2000.

3 Hunt SA, Baker DW, Chin MH, et al. ACC/AHA guidelines for the evaluation and management of chronic heart failure in the adult. J Am Coll Cardiol. 2001;38:2101-2113.

4 Gheorghiade M, Bonow BO. Chronic heart failure in the United States: a manifestation of coronary artery disease. Circulation. 1998;97:282-289.

5 Schoeller R, Andresen D, Buttner P, et al. First- or second-degree atrioventricular block as a risk factor in idiopathic dilated cardiomyopathy. Am J Cardiol. 1993;71:720-726.

6 Aaronson K, Schwartz J, Chen TM, et al. Development and prospective validation of a clinical index to predict survival in ambulatory patients referred for cardiac transplant evaluation. Circulation. 1997;95:2660-2667.

7 Brecker SJ, Xiao HB, Sparrow J, et al. Effects of dual-chamber pacing with short atrioventricular delay in dilated cardiomyopathy. Lancet. 1992;340:1308-1312.

8 Grines CL, Bashore TM, Boudoulas H, et al. Functional abnormalities in isolated left bundle branch block: the effects of interventricular asynchrony. Circulation. 1989;79:845-853.

9 Farwell D, Patel NR, Hall A, et al. How many people with heart failure are appropriate for biventricular resynchronization? Eur Heart J. 2000;21:1246-1250.

10 Iuliano S, Fischer SG, Karasik PE, et al., for the Department of Veterans Affairs Survival Trial of Antiarrhythmic Therapy in Congestive Heart Failure. QRS duration and mortality in patients with congestive heart failure. Am Heart J. 2002;143:1085-1091.

11 Saxon LA, Kumar UN, DeMarco T. Heart failure and cardiac resynchronization therapies: US experience in the year 2000. Ann Noninvasive Electrocardiol. 2000;5(2):188-194.

12 Salukhe TV, Francis DP, Sutton R. Comparison of medical therapy, pacing, and defibrillation in heart failure (COMPANION) trial terminated early; combined biventricular pacemaker-defibrillators reduce all-cause mortality and hospitalization. Int $J$ Cardiol. 2003;87:119-120.

13 Auricchio A., Stellbrink C, Black M, et al. Effect of pacing chamber and atrioventricular delay on acute systolic function of paced patients with congestive heart failure. The Pacing Therapies for Congestive Heart Failure Study Group. The Guidant Congestive Heart Failure Research Group. Circulation. 1999;99:2993-3001.

14 Thackray S, Coletta A, Jones $P$, et al. Clinical trails update: highlights of the Scientific Sessions of Heart Failure 2001, a meeting of the Working Group on Heart Failure of the European Society of Cardiology. CONTAK-CD, CHRISTMAS, OPTIME-CHF. Eur $J$ Heart Fail. 2001;3:491-494.

15 Abraham WT, Fisher WG, Smith AL, et al. Cardiac resynchronization in chronic heart failure: The MIRACLE study. N Engl J Med. 2002;346(24):1845-1853.

16 Cleland JGF, Daubert JC, Erdmann E, et al. The CARE-HF study (Cardiac Resynchronization in Heart Failure study): rationale, design and end-points. Eur J Heart Fail. 2001;3:481-489.

17 Cazeau S, Leclercq C, Lavergne T, et al. Effects of multisite biventricular pacing in patients with heart failure and intraventricular conduction delay. N Engl J Med. 2001;344:873-880.

18 Sawhney N, Faddis M. Resynchronization therapy for congestive heart failure. Curr Treat Options Cardiovasc Med. 2000;4:277-285.

19 Cardiac Resynchronization Therapy, Mechanisms and Device Trials. Minneapolis, MN: Medtronic, Inc.; 2003.

20 Cardiac Resynchronization Therapy for Heart Failure Management. St. Paul, MN: Guidant Corp; 2003. 